\title{
Extubação não programada em crianças internadas em UTI: incidência e fatores associados
}

Unscheduled extubation in children admitted to the ICU: incidence and associated factors

Extubación no programada en niños ingresados en UCl: incidencia y factores associados

Leslie Sue Fernandes ${ }^{1}$ (D) https://orcid.org/0000-0001-9702-8699

Carmina Silva dos Santos ${ }^{1}$ (D) https://orcid.org/0000-0002-0101-3546

Claudiane Maria Urbano Ventura ${ }^{1}$ (D) https://orcid.org/0000-0003-0079-4878

\section{Resumo}

Objetivo: Investigar a incidência e os fatores de riscos associados à extubação não programada em crianças internadas nas Unidades de Terapia Intensiva Neonatal e Pediátrica de um hospital escola de referência em Recife - PE.

Métodos: Estudo prospectivo realizado com crianças intubadas, entre maio e agosto de 2013. Utilizou-se um formulário estruturado e os dados foram obtidos dos prontuários e analisados através dos softwares SPSS 13.1 e Stata 12.1.

Resultados: Foi identificado uma incidência de 10,9 extubações não programadas por 100 intubados-dia. Logo foram implementadas medidas para redução da ocorrência desse evento e após cinco anos verificado novamente sua incidência. Uma redução de $60 \%$, nos casos de extubações, foi observada.

Conclusão: A alta incidência de extubações não programadas encontrada nesse estudo e, posteriormente sua redução com as intervenções de melhorias, reforça a necessidade de educação continuada da equipe multiprofissional.

\section{Abstract}

Objective: To investigate the incidence and risk factors associated with unscheduled extubation in children admitted to the Neonatal and Pediatric Intensive Care Units of a reference teaching hospital in Recife - PE.

Methods: Prospective study carried out with intubated children, between may and august 2013. A structured form was used and data were obtained from medical records and analyzed using SPSS 13.1 and Stata 12.1 software. Results: An incidence of 10.9 unscheduled extubations per 100 intubated days was identified. Measures were soon implemented to reduce the occurrence of this event and after five years its incidence was checked again. A $60 \%$ reduction in extubation cases was observed.

Conclusion: The high incidence of unscheduled extubations found in this study and, subsequently, its reduction with improvement interventions, reinforces the need for continuing education of the multidisciplinary team.

\section{Resumen}

Objetivo: Investigue la incidencia y los factores de riesgo asociados con la extubación no programada en niños hospitalizados en las Unidades de Cuidados Intensivos Neonatales y Pediátricos de un hospital escolar de referencia en Recife - PE

Métodos: Estudio prospectivo realizado con niños intubados, entre mayo y agosto de 2013. Se utilizó un formulario estructurado y los datos se obtuvieron de las historias clínicas y se analizaron mediante el software SPSS 13.1 y Stata 12.1

Resultados: Se identificó una incidencia de 10,9 extubaciones no programadas por 100 días intubados. Pronto se implementaron medidas para reducir la ocurrencia de este evento y luego de cinco años se volvió a verificar su incidencia. Se observó una reducción del $60 \%$ en los casos de extubación.

Conclusión: La alta incidencia de extubaciones no programadas encontrada en este estudio y, posteriormente, su reducción con intervenciones de mejora, refuerza la necesidad de formación continua del equipo multidisciplinario.

\section{Como citar:}

Fernandes LS, Santos CS, Ventura CM. Extubação não programada em crianças internadas em UTI: incidência e fatores associados. Rev Soc Bras Enferm Ped. 2021;21(1):37-43.

${ }^{1}$ Instituto de Medicina Integral Prof. Fernando Figueira, Recife, PE, Brasil.

Conflitos de interesse: nada a declarar.

Submetido: 9 de Março de 2021 | Aceito: 30 de Julho de 2021

Autor correspondente: Leslie Sue Fernandes | E-mail: leh_sue@hotmail.com

DOI: http://dx.doi.org/10.31508/1676-3793202100007 


\section{Introdução}

Com o desenvolvimento e aperfeiçoamento de novas técnicas de atendimento a criança criticamente enferma, a ventilação mecânica invasiva, através de um tubo endotraqueal (TET), surge com a finalidade de otimizar a troca gasosa e a administração de oxigênio naqueles que não conseguem manter por si próprio sua função respiratória satisfatoriamente e de forma espontânea, contribuindo para o aumento da sobrevida. ${ }^{(1-3)}$

No entanto, eventos adversos relacionados à assistência ventilatória são frequentes e representam riscos adicionais a segurança dos pacientes. ${ }^{4-}$ 6) A extubação não programada (ENP) destaca-se, uma vez que está entre os eventos mais descritos nas unidades de terapia intensiva (UTI), e o principal relacionado à ventilação mecânica invasiva (VMI). ${ }^{(4,5,7,8)}$

A ocorrência de ENP trás conseqüências em diversos aspectos aos pacientes; os expõem a complicações da intubação endotraqueal de emergência quando uma reintubação é necessária, e possivelmente, aumento do tempo de VMI e permanência na UTI. ${ }^{(9)}$

Melhorar a qualidade da assistência e garantir a segurança do paciente, atualmente, tem sido uma prioridade na área da saúde, principalmente quando se refere a ambientes extremamente complexos no âmbito hospitalar como são as unidades de terapia intensiva neonatal e pediátrica. ${ }^{(10)}$

A equipe de enfermagem tem um papel relevante nesse cenário já que assume o cuidado de assistência direto ao paciente de forma contínua. Portanto, cabe ao enfermeiro além do cuidado ao paciente, a supervisão da equipe multiprofissional para obter-se uma assistência livre de danos. ${ }^{(10,11)}$

Sabendo que eventos adversos são passíveis de prevenção, é imprescindível inicialmente identificá-los a fim de estabelecer estratégias com o intuito de minimizar sua ocorrência. Desta forma, foi desenvolvido esse estudo com o objetivo de investigar a incidência e os fatores de riscos associados à extubação não programada em crianças internadas nas unidades de terapia intensiva neonatal e pediátrica de um hospital escola de referência.

\section{Métodos}

Trata-se de um estudo de coorte prospectivo com abordagem quantitativa, realizado nas Unidades de Terapia Intensiva Neonatal (UTIN) e Pediátrica (UTIP) de um hospital escola de referência, localizado em Recife (PE), reconhecido como uma das estruturas hospitalares mais importantes do país e centro de referência assistencial materno-infantil.

A amostra dessa pesquisa foi composta por todas as crianças admitidas nas unidades supracitadas, no período de maio a agosto de 2013, e que foram submetidas ao procedimento de intubação endotraqueal para suporte ventilatório em algum momento do internamento na unidade.

Foram excluídos da pesquisa: recém-nascidos intubados exclusivamente para finalidade terapêutica com surfactante, e que foram extubados logo após a administração da droga e pacientes com menos de 24 horas de internamento nas unidades.

Para determinar o tamanho da amostra, foi observado por 30 dias antes da coleta de dados, a ocorrência de intubações traqueais e ENP em ambas as unidades. Uma amostra de 1.371 crianças intubadas-dia foi calculada para estimar a taxa de extubação não programada, com erro de estimação não superior a 1.0, para mais ou para menos, usando um intervalo de confiança de $95 \%$, admitindo que a taxa esperada seja de cinco ENP por 100 crianças intubadas-dia.

Os dados foram coletados através de visitas diárias na UTIN e UTIP em busca das admissões. Sendo identificado o paciente elegível, foram convidados os pais ou responsáveis legais da criança a participarem da pesquisa e solicitado a assinatura do Termo de Consentimento Livre e Esclarecido (TCLE).

As crianças incluídas no estudo foram acompanhadas durante toda sua permanência na UTI, independentemente do número de vezes que precisaram ser intubadas. Foi realizada revisão prospectiva dos prontuários em busca dos registros dos profissionais envolvidos na assistência do paciente. A informação sobre a ocorrência da extubação não programada também foi obtida através do livro de registro de eventos adversos.

Utilizou-se um formulário estruturado contendo questões abertas e fechadas elaborado pelas próprias 
pesquisadoras, com as variáveis do estudo, de forma a garantir o alcance dos objetivos propostos. Com a finalidade de verificar a adequação e a forma de aplicação do instrumento, foi realizado um teste piloto e nenhum ajuste foi necessário.

Os dados obtidos foram digitados no Microsoft Excel e analisados softwares SPSS 13.1 e Stata 12.1. No primeiro momento foi realizada uma análise descritiva a fim de estudar o comportamento das variáveis, sendo resumidos em frequência absoluta e relativa e apresentados em tabelas. Para as variáveis categóricas usou-se o teste qui-quadrado de tendência; para as variáveis quantitativas, utilizou-se o teste de Mann-Whitney, calculando-se a mediana.

Foram ajustados modelos de Poisson para comparar, através da razão de risco, a frequência do desfecho entre as seguintes variáveis: gênero, idade, peso, tipo de internamento e tempo de intubação. As variáveis que, nessa análise, mostraram-se associadas ao desfecho, com nível de significância observado menor ou igual a $20 \%$, (valor $\mathrm{p}<0,20)$ foram selecionadas para a análise multivariada. Para a obtenção do modelo final, utilizou-se o procedimento backward (passo a passo para trás). Em cada passo, eliminava-se a variável que apresentava o maior valor $p$ acima de 0,05 . Ao final do procedimento apenas uma variável compôs o modelo final, com valor $\mathrm{p}<0,05$.

O método de Kaplan-Meier foi utilizado para analisar a ocorrência de ENP com o tempo de intubação. Deve-se esclarecer que para essa análise foi considerada a ocorrência do primeiro evento de cada paciente. Para todos os testes foi adotado um nível de significância de $5 \%$.

O estudo recebeu previamente a aprovação do comitê de ética em pesquisa da própria instituição, sob o parecer n.3519-13, conforme a Resolução n.466/12 do Ministério da Saúde. ${ }^{(12)}$

\section{Resultados}

A coorte foi constituída de 139 participantes, dos quais obteve-se 294 intubações traqueais, sendo apenas uma por via nasal. Foram observados 129 episódios de ENP, resultando em uma densidade de incidência de 10,9 eventos por 100 pacientes intubados-dia. $\mathrm{Na}$ tabela 1 estão descritas as características da amostra conforme a ocorrência de ENP. Nela pode-se verificar que as variáveis: idade, peso, tipo de internamento, egresso e tempo de permanência na UTI obtiveram associação estatística com o desfecho $(\mathrm{p}<0,001)$.

Tabela 1. Distribuição das características da amostra conforme a ocorrência de extubação não programada nas Unidades de Terapia Intensiva Neonatal e Pediátrica

\begin{tabular}{|c|c|c|c|}
\hline \multirow[b]{2}{*}{ Característica } & \multicolumn{2}{|c|}{ Extubação não programada } & \multirow[b]{2}{*}{$p$-value } \\
\hline & $\underset{(n=65)}{\operatorname{Sim}}$ & $\begin{array}{c}\text { Não } \\
(n=74)\end{array}$ & \\
\hline Gênero $^{\dagger \dagger}$ & & & $0,609^{*}$ \\
\hline Feminino & $31(44,9)$ & $38(55,1)$ & \\
\hline Idade $\mathrm{e}^{\dagger \dagger}$ & & & $<0,001^{*}$ \\
\hline Recém-nascido & $52(62,7)$ & $31(37,3)$ & \\
\hline Lactente & $12(28,6)$ & $30(71,4)$ & \\
\hline Acima de 2 anos & $1(7,1)$ & $13(92,9)$ & \\
\hline Peso, $\mathrm{kg}^{\dagger}$ & $\begin{array}{c}1,14(0,88- \\
2,41)\end{array}$ & $\begin{array}{c}4,15(2,41- \\
8,90)\end{array}$ & $<0,001^{* *}$ \\
\hline Tipo de internamento ${ }^{\dagger \dagger}$ & & & $<0,001^{*}$ \\
\hline Clínico & $55(64,7)$ & $30(35,3)$ & \\
\hline Cirúrgico & $10(18,5)$ & $44(81,5)$ & \\
\hline Egresso da UTI ${ }^{\dagger \dagger}$ & & & $<0,001^{*}$ \\
\hline Alta melhorada & $6(12,2)$ & $43(87,8)$ & \\
\hline $\begin{array}{l}\text { Transferência para outra UTI } \\
\text { ou UCl }\end{array}$ & $45(68,2)$ & $21(31,8)$ & \\
\hline Óbito & $14(58,3)$ & $10(41,7)$ & \\
\hline Tempo de VMI, (dias) $^{\dagger}$ & $3,0(1,0-6,0)$ & $\begin{array}{c}4,0(2,0- \\
8,0)\end{array}$ & $0,238^{* *}$ \\
\hline $\begin{array}{l}\text { Tempo de permanência na UTI, } \\
\text { (dias) }^{\dagger}\end{array}$ & $\begin{array}{c}16,0(9,0- \\
35,0)\end{array}$ & $\begin{array}{c}6,0(3,0- \\
11,0)\end{array}$ & $<0,001^{* *}$ \\
\hline
\end{tabular}

${ }^{\dagger \dagger}$ Frequência simples (percentagem); † Mediana (Q1 - Q3); " Teste qui-quadrado; ${ }^{* *}$ Teste de Mann-Whitney

A tabela 2 apresenta as características dos eventos ocorridos, onde é percebido que a maior prevalência de ENP ocorreu de forma mecânica, tendo a predominância de obstrução do TET por secreções $(47,2 \%)$ e assicronia do ventilador mecânico com queda da saturação de oxigênio, sem obstrução do tubo (38,8\%). É possível observar ainda que $84,5 \%$ dos que sofreram o evento não estavam em processo de desmame da ventilação mecânica e necessitaram, consequentemente, ser reintubados $(80,6 \%)$.

Através do teste de Kaplan-Meier (Figura 1) foi constatado uma relação inversamente proporcional entre a probabilidade dos pacientes intubados livres de ENP e o tempo de permanência com TET. Observa-se que no primeiro, quinto e décimo dia a probabilidade dos pacientes não apresentarem ENP foram, respectivamente, 59,3\%, 20,3\% e 8,5\%, demonstrando o aumento do risco de extubação com o decorrer do tempo de exposição. 
Tabela 2. Distribuição de frequência e percentagem das características das extubações não programadas ocorridas nas Unidades de Terapia Intensiva Neonatal e Pediátrica

\begin{tabular}{lc}
\hline Variáveis & $\mathrm{n}(\%)$ \\
\hline Tipo da ENP & \\
Acidental & $57(44,19)$ \\
Mecânica & $72(55,81)$ \\
Período da ENP & \\
07 às 13 horas & $42(32,56)$ \\
13 às 19 horas & $47(36,43)$ \\
19 às 07 horas & $40(31,01)$ \\
Secreção em vias aéreas & \\
Pouca quantidade & $27(20,93)$ \\
Moderada quantidade & $61(47,29)$ \\
Grande quantidade & $41(31,78)$ \\
Uso de sedação & \\
Sim & $22(17,05)$ \\
Não & $107(82,95)$ \\
Uso de restrição física & \\
Sim & $4(3,10)$ \\
Não & $125(96,90)$ \\
Desmame da VM & \\
Sim & $20(15,50)$ \\
Não & $109(84,50)$ \\
Reintubação & \\
Sim & $104(80,62)$ \\
Não & $25(19,38)$ \\
\hline n(\%) - Frequência simples (percentagem) &
\end{tabular}

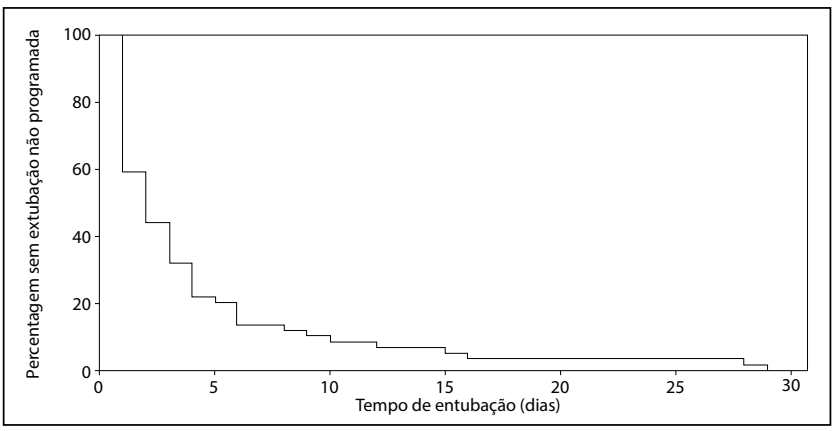

Figura 1. Curva de Kaplan-Meier com estimativas da proporção de pacientes sem extubação não programada nas Unidades de Terapia Intensiva Neonatal e Pediátrica
A tabela 3 apresenta os resultados das análises univariada e multivariada de fatores associados à ENP entre as crianças estudadas. Na análise univariada, as variáveis idade, peso, tipo de internamento e tempo de intubação apresentaram $\mathrm{p}<0,20$. Entretanto, quando realizado a análise multivariada apenas o tempo de intubação apresentou associação significante com o desfecho $(\mathrm{p}<0,05)$.

\section{Discussão}

Nos estudos avaliando a qualidade na assistência à saúde, a ENP tem sido um dos eventos adversos mais descritos devido à sua alta frequência nas UTIs. ${ }^{(7,8)}$ Entre crianças, encontram-se taxas de incidência que variam de 0,41 a 5,34 ENP por 100 pacientes intubados-dia. ${ }^{(9,13-16)}$

Na coorte desse estudo foi encontrada uma incidência de ENP de 10,9, superior às relatadas na literatura. A esse resultado deve-se levar em consideração as características do local e da população estudada: hospital escola de referência em pediatria e recém-nascidos prematuros de extremo baixo peso e formador de equipes multiprofissionais.

Não há consenso descrito acerca da incidência aceitável de ENP em UTIN e UTIP já que existem poucos estudos na literatura que avaliam tal fato. Uma taxa próxima a zero parece ser o desejável, mas ao mesmo tempo arriscado, uma vez que seria necessário utilizar altas doses de sedação ou realizar extubações antes que os pacientes atendam os critérios ideais de desmame da VMI; estas situações também estarão relacionadas a uma má evolução clínica. ${ }^{(13-16)}$

Tabela 3. Resultados das análises uni e multivariada, com ajustes de modelos de Poisson, para a ocorrência de extubação não programada nas Unidades de Terapia Intensiva Neonatal e Pediátrica

\begin{tabular}{|c|c|c|c|c|c|c|}
\hline \multirow[t]{2}{*}{ Variáveis } & \multirow{2}{*}{$\begin{array}{c}\text { Amostra } \\
\mathrm{n}\end{array}$} & \multirow{2}{*}{$\begin{array}{c}\text { Desfecho } \\
n(\%)\end{array}$} & \multicolumn{2}{|c|}{ Análise univariada } & \multicolumn{2}{|c|}{ Modelo final } \\
\hline & & & $\mathrm{RR}(\mathrm{IC95 \% )}$ & p-value & $\mathrm{RR}(\mathrm{IC} 95 \%)$ & p-value \\
\hline Tipo de internamento & & & & $<0,001$ & & 0,813 \\
\hline Clínico & 85 & $55(64,7)$ & $3,5(1,95-6,26)$ & & $1,1(0,47-2,56)$ & \\
\hline Cirúrgico & 54 & $10(18,5)$ & 1,0 & & 1,0 & \\
\hline Gênero & & & & 0,611 & - & - \\
\hline Feminino & 69 & $31(44,9)$ & 1,0 & & - & \\
\hline Idade & & & & 0,001 & & 0,625 \\
\hline Recém-nascido & 83 & $52(62,7)$ & $2,7(1,63-4,48)$ & & $1,1(0,70-1,81)$ & \\
\hline Lactente ou mais & 56 & $13(23,2)$ & 1,0 & & 1,0 & \\
\hline Peso (kg) & 139 & - & $0,9(0,77-1,02)$ & 0,100 & $1,0(0,94-1,06)$ & 0,924 \\
\hline Tempo de intubação & 139 & - & $1,02(1,01-1,03)$ & $<0,001$ & $1,02(1,00-1,04)$ & 0,002 \\
\hline
\end{tabular}

IC95\% - Intervalo de 95\% de confiança 
De uma forma geral, a ENP é definida como qualquer extubação inesperada ou realizada em momento não planejado, podendo ser dividida em acidental, mecânica e auto-extubação. ${ }^{(17)}$ Percebe-se na literatura que a maior parte das extubações em crianças foram acidentais, devido a agitação do paciente, fixação e posicionamento inadequado do TET e manuseio para procedimentos e cuidado; $10 \%$ foi do tipo mecânica por obstrução do dispositivo e outros $10 \%$ à auto-extubação devido a ineficiência da contenção e/ou maior nível de consciência. ${ }^{(6,13,14,16,18)}$

Diferindo, nesta pesquisa observou-se que a maior parte das ENP foi do tipo mecânica $(55,8 \%)$ devido, principalmente, a obstrução do TET. Deve-se ressaltar que $79,1 \%$ dos pacientes estudados foram registrados com moderada a grande quantidade de secreções em vias aéreas, o que pode estar relacionado diretamente com o alto percentual deste evento. As extubações acidentais contribuíram com $44,2 \%$, porém não foi possível especificar a causa pois não foram encontradas informações nos prontuários.

Os estudos acerca desse evento adverso vêm apontando como principais fatores para a sua ocorrência a idade do paciente, nível de sedação, fixação do TET, quantidade de secreções em vias aéreas, via de intubação endotraqueal, causa do internamento na UTI, contenção física e procedimentos de cuidados. ${ }^{13-}$ ${ }^{15)}$ Os resultados observados na amostra desse trabalho corroboram com os achados da literatura, pois encontraram-se evidências semelhantes.

No atual estudo observa-se que a frequência de ENP foi significativamente maior quanto menor a idade e o peso dos sujeitos, atingindo de maneira expressiva os neonatos. Entretanto, tais variáveis não permaneceram significativas na análise múltipla, embora exista uma tendência de risco $(p=0,001$ e 0,100).

Possivelmente o curto comprimento da traqueia, a menor área de superfície corpórea disponível para fixação do tubo e por permanecerem mais tempo em VMI, são fatores que favorecem os recém-nascidos a maior ocorrência das ENP.(15) Além disso, a redução do uso de sedação, à ausência de restrições físicas, o aumento do número de procedimentos realizados e o perfil de manipulação da equipe multiprofissional nessa população também contribuem. ${ }^{(18)}$

Na amostra estudada encontrou-se um percentual baixo daqueles que usaram sedação (17\%) e restrição física (3\%). O uso desses métodos na UTI ainda é controverso, sendo necessário que a equipe multidisciplinar discuta a melhor forma para o paciente, para que não fiquem acordados e em VMI desnecessariamente, com possibilidade de ENP e complicações associadas ao evento. ${ }^{(14,18)}$

Em relação ao sexo dos estudados, não foram encontradas diferenças estatísticas entre os que sofreram a ENP quando comparados àqueles que não sofreram este episódio $(p=0,609)$. Estudos relacionados demonstraram não haver relação entre sexo e a ocorrência do evento. ${ }^{(15,18)}$

Foram encontrados no perfil da amostra predominância dos internamentos clínicos em relação aos cirúrgicos, como relatados em outra pesquisa. ${ }^{(19)}$ Os problemas respiratórios têm ocupado as primeiras posições de internamento clínico nas UTIN e UTIP, enquanto as anomalias congênitas, como as cardiopatias, os internamentos cirúrgicos. ${ }^{(20)}$

A criança é particularmente suscetível a desenvolver doenças do sistema respiratório, pois existem diversos fatores inter-relacionados que favorecem essa evolução, que vão desde peculiaridades anatômicas a características fisiológicas. ${ }^{(19)}$ Essa situação faz com que permaneçam longos períodos em uso da VMI na UTI, o que favorece o maior risco de ENP, diferentes dos casos cirúrgicos que usam a VMI para o pós-operatório, diminuindo os episódios do evento, estatisticamente comprovados nesta pesquisa $(p<0,001)$.

Estudos atuais têm apontado que é no período diurno que vem ocorrendo a maioria das ENP, atribuídos pela maior frequência de procedimentos, como a avaliação dos profissionais, coleta de sangue, punção lombar, higiene corporal, radiografias, mudança de decúbito, entre outros. ${ }^{(13-16,18,21)}$ Nas unidades estudadas foi encontrada que aproximadamente $70 \%$ das ENP ocorreram durante o dia, compatível com o tempo em que os cuidados aos pacientes são intensificados. Assim, toda a equipe multiprofissional deve estar atenta à frequência e à execução desses manuseios, sendo orientada e preparada para prevenir a ocorrência de ENP. ${ }^{(15)}$ Os eventos ocorridos no período noturno estão relacionados, provavelmente, ao número reduzido da equipe assistencial e, consequentemente, a menor vigilância dos internados..$^{(13,18,21)}$

Alguns estudos recentes com recém-nascidos têm demonstrado que o tempo prolongado de VMI, au- 
menta o risco para a ENP, sendo esse o preditor isolado mais significativo para ocorrência do evento. ${ }^{(13-15)}$ Foi observado ainda que a cada dia de intubação o risco de ENP aumentava 2 a 3\%, sendo nos primeiros dez dias de VMI a maior taxa de ENP $(79,6 \%)^{(9,13,15)}$

Esse mesmo fator foi encontrado no presente estudo conforme o modelo final de Poisson $(\mathrm{p}=0,002)$ bem como através do método de Kaplan Méier onde foi demonstrando que o risco de ENP aumentou com o tempo de uso do TET, ocorrendo significativamente já nos primeiros cinco dias de intubação. Uma possível hipótese para explicar tal achado é que nos primeiros dias de internamento na UTI os pacientes são submetidos a vários procedimentos para diagnósticos, o que consequentemente favorece a maior ocorrência do evento adverso nesse período. ${ }^{(14)}$ Para redução da ENP esta informação tem grande importância a fim de aumentar a atenção durante a manipulação dos pacientes.

Deve-se apontar que quase $85 \%$ dos que sofreram o evento não estavam em processo de desmame da ventilação mecânica precisando, consequentemente, ser reintubados. Estudo atual mostrou resultado semelhante com taxas de reintubação acima de $80 \%$, uma vez que os pacientes eram incapazes de realizar ventilação espontânea fora do VMI após a ENP devido a sua condição clínica ou nível inadequado de sedação, sendo expostas as complicações desse procedimento. ${ }^{(9)}$

Uma revisão sistemática com recém-nascidos relata que a reintubação após uma ENP, além de todo estresse causado ao paciente e à equipe assistencial, pode estar associada a diversas complicações como broncoaspiração, hipóxia, atelectasia, trauma de vias aéreas, lesão pulmonar por ventilação excessiva, aumento do risco de pneumonia associada a VMI, hemorragia intracraniana, instabilidade hemodinâmica, aumento do tempo de uso de VMI, arritmia, parada cardiorrespiratória e morte. ${ }^{(6)}$

Assim sendo, a ENP é um evento adverso grave que pode interferir na integridade do paciente causando importantes consequências tanto no que se refere ao sofrimento desnecessário como no aumento da dor, aumento do risco de infecções hospitalares e prolongamento do tempo de internamento na UTI.

Uma pesquisa mostrou que o tempo de permanência hospitalar aumentou em $11 \%$ naqueles que apresentaram a ENP. ${ }^{(18)}$ Outro estudo mostrou que os que sofreram o evento tiveram uma média de interna- mento na UTIP de 9,5 dias quando comparados aos que não sofreram, com 7 dias $(\mathrm{p}=0,049)$. $^{(13)}$

Análogo a isso, no presente estudo foi encontrado que pacientes com ENP tiveram maior tempo médio de permanência na UTI (16 dias versus 6 dias, $\mathrm{p}<0,001$ ). Como alguns estudos, neste não foi observado nenhum óbito relacionado diretamente a ENP, porém foi constatado que o grupo que sofreu o evento apresentou mais óbitos do que os que não sofreram $(\mathrm{p}<0,001)$. . $^{(13,14)}$

Os resultados expostos e a alta incidência encontrada nesse estudo gerou grande inquietação entre os profissionais envolvidos. Desta forma, os gestores dos setores incluídos na pesquisa uniram forças, a fim de construir e implementar estratégias direcionadas à melhoria da assistência aos menores internados em UTI e consequentemente redução da incidência de ENP.

Semelhante a estudo recente, novas medidas foram associadas ao atendimento nas UTIs, em conjunto constante com a Educação Permanente da Instituição. ${ }^{(22)}$ Tais medidas incluíram, uso de bandagem específica para fixação do TET, inclusão de suportes para apoiar os circuitos do VMI, a fim de não haver tração do mesmo e melhorar a posição do TET, cuidados prestados ao paciente em duplas, treinamento para aspiração das vias aéreas, medidas de controle da dor e início do protocolo de manuseio mínimo para os recém-nascidos prematuros.

Após cinco anos da implementação dessas medidas, foi feito novamente o levantamento da ocorrência de ENP através dos formulários de eventos adversos presentes na UTIN e UTIP, que são preenchidos diariamente. Satisfatoriamente, foi encontrado uma incidência de ENP de 4,5 eventos por 100 pacientes intubados-dia; percebe-se uma redução dessa taxa da pesquisa inicial em quase $60 \%$. Outra pesquisa comparativa também mostrou essa mudança com a implantação de novas estratégias de cuidados. ${ }^{(23)}$

Observou-se ainda, que dessas ENP, 68,2\% ocorreram de forma acidental e pouco mais da metade (56,8\%) necessitaram ser reintubados. Possivelmente, a maior parte desse evento deve ter ocorrido durante o período de programação para a extubação planejada e desmame da sedação, havendo assim uma menor quantidade de reintubação. Isso também diferiu do resultado inicial onde foi obtido taxas de reintubação de $80 \%$, uma vez que, quase a totalidade das ENP ocorreram fora do processo de desmame da VMI. 
Apesar de não ter desenhado um outro estudo prospectivo para avaliar a eficácia das intervenções implantadas, é possível notar claramente o êxito de um programa de melhoria para a redução da incidência de ENP.

Algumas limitações foram encontradas no decorrer dessa pesquisa. Primeiro, a dificuldade de obter as assinaturas do TCLE pelos responsáveis pela criança, tanto pela ausência deles durante o internamento ou mesmo pelo medo de participar do estudo, o que resultou em uma diminuição da amostra. Segundo o desenho do estudo compreendeu coleta de dados secundários e, por vezes, encontrou-se registros incompletos da ocorrência da ENP pelos profissionais envolvidos, ocasionando perdas de dados.

Deve-se salientar a escassez de dados na literatura sobre ENP entre crianças, principalmente quando se discute acerca de estratégias para redução da ocorrência desse evento. Sendo assim, esse trabalho apresenta relevância clínica, uma vez que contribui para o conhecimento e a discussão dessa temática.

\section{Conclusão}

A alta incidência de ENP encontrada nesse estudo e, posteriormente a sua redução com as intervenções de melhorias, reforça a necessidade de educação continuada da equipe multiprofissional para conscientizá-los e capacitá-los constantemente. É fundamental a implantação de protocolos assistenciais com a finalidade de sistematizar as condutas e técnicas nos procedimentos, com vista a melhoria da qualidade da assistência. Espera-se que os resultados revelados nessa pesquisa contribuam para identificar precocemente os pacientes suscetíveis a ENP e desenvolver ações para a melhoria da assistência em saúde, a fim de proporcionar segurança dos menores internados na UTI.

\section{Referências}

1. Piva JP, Amantéa S, Luchese S, Giugno K, Maia TR, Einloft L. Accidental Extubation in a Pediatric Intensive Care Unit. J Pediatr (Rio J). 1995;71(2):72-6.
2. Gomes GP, RezendeAA,Almeida JD, Silva IL, Beresford H. Nursing care for patients with orotracheal tube: evaluation performed at intensive care unit. Rev Enferm UFPE on line. 2009;3(4):20-5.

3. Chang LC, Liu PF, Huang YL, Yang SS, Chang WY. Risk factors associated with unplanned endotracheal selfextubation of hospitalized intubated patients: a 3-year retrospective case-control study. Appl Nurs Res. 2011;24(3):188-92.

4. Kapadia FN, Bajan KB, Raje KV. Airway accidents in intubated intensive care unit patients: an epidemiological study. Crit Care Med. 2000;28(3):659-64.

5. Chen CM, Chan KS, Fong Y, Hsing SC, Cheng AC, Sung MY, et al. Age is an important predictor of failed unplanned extubation. Int I Gerontol. 2010;4(3):120-9.

6. Silva PS, Fonseca MC. Unplanned endotracheal extubations in the intensive care unit: systematic review, critical appraisal, and evidence-based recommendations. Anesth Analg. 2012;114(5):1003-14.

7. Ventura CM, Alves JG, Meneses JA. Eventos adversos em Unidade de Terapia Intensiva Neonatal. Rev Bras Enferm. 2012;65(1):49-55.

8. Cossul MU, Neiva LE, Silveira A0. Notificação de eventos adversos em uma unidade de terapia intensiva neonatal. Rev Enferm UFPE on line. 2021;15:e246969.

9. Mattos MC, Silva GA, Andreazza MG, Rodrigues FS, Oliveira IC, Cat MN. Prevalência de extubação não planejada e fatores associados em uma unidade de terapia intensiva neonatal. Rev Pesqui Fisioter. 2020;10(3):442-50.

10. Gaíva MA, Souza JS, Xavier JS. A segurança do paciente em unidade de terapia intensiva neonatal: uma revisão de literatura. Rev Enferm UFPE on line. 2013;7(Esp):928-36.

11. Gaíva MA, Rondon JN, Jesus LN. Segurança do paciente em unidade de terapia intensiva neonatal: percepção da equipe de enfermagem. Rev Soc Bras Enferm Ped. 2017;17(1):14-20.

12. Brasil. Ministerio da Saúde. Conselho Nacional de Saúde. Resolução No. 466 de 12 de dezembro de 2010. Brasília (DF): Ministério da Saúde; 2010. [citado 2019 Ago 20]. Disponível em: http://bvsms.saude.gov.br/bvs/saudelegis/cns/2013/ res0466_12_12_2012.html

13. Meregalli CN, Baron FA, D’Alessandro MA, Danzi EP, Debaisi GE. Impacto de una intervención de mejora de calidad sobre la incidencia de extubaciones no planeadas em una unidad de cuidados intensivos pediátricos. Arch Argent Pediatr. 2013;111(5):391-7.

14. Oliveira PC, Cabral LA, Schettino RC, Ribeiro SN. Incidência e principais causas de extubação não planejada em unidade de terapia intensiva neonatal. Rev Bras Ter Intensiva. 2012;24(3):230-35.

15. Carvalho FL, Mezzacappa MA, Calil R, Machado HC. Incidência e fatores de risco para a extubação acidental em uma unidade de terapia intensiva neonatal. J. Pediatr (Rio J). 2010;86(3):189-95.

16. Merkel L, Beers K, Lewis MM, Stauffer J, Mujsce D, Kresch M. Reducing unplanned extubations in the NICU. Pediatrics. 2014;133(5):1367-72.

17. Lucchini A, Bambi S, Galazzi A, Elli S, Negrini C, Vaccino S, et al. Unplanned extubations in general intensive care unit: a nine-year retrospective analysis. Acta Biomed. 2018;89(7-S):25-31.

18. Pontes LF, Gardenghi G, Capucho HC. Caracterização de casos de extubação acidental em pacientes assistidos em hospitais universitários federais. Fisioter Pesqui (Online). 2017;7(4):531-7.

19. Lanetzki CS, Oliveira CA, Bass LM,Abramovici S, Troster El. 0 perfil epidemiológico do Centro de Terapia Intensiva Pediátrico do Hospital Israelita Albert Einstein. Einstein (São Paulo). 2012;10(1):16-21.

20. Mendonça JG, Guimarães MJ, Mendonça VG, Portugal JL, Mendonça CG. Perfil das internações em Unidades de Terapia Intensiva Pediátrica do Sistema Único de Saúde no estado de Pernambuco, Brasil. Ciênc Saúde Colet. 2019;24(3):907-16.

21. Souza RF, Alves AS, Alencar IG. Eventos adversos na unidade de terapia intensiva. Rev Enferm UFPE on line. 2018;12(1):19-27.

22. Torres GM, Nascimento ER, Hermida PM, Malfussi LB, Galetto SG. Care for unplanned extubation prevention: analysis of the validity of an instrument's content. Rev Bras Enferm. 2021;74(1):e20180998.

23. Chao CM, Lai CC, Chan KS, Cheng KC, Ho CH, Chen CM, et al. Multidisciplinary interventions and continuous quality improvement to reduce unplanned extubation in adult intensive care units: A 15-year experience. Medicine (Baltimore). 2017;96(27):e6877. 